\title{
Colorado's Advanced Materials Institute Holds Second Conference
}

Colorado's Advanced Materials Institute (AMI) held its second Industry-University Advanced Materials Conference in Denver, March 6-9, 1989. Chaired by Fred Smith (Colorado State University) and cosponsored by the Materials Research Society and the Colorado Advanced Technology Institute, the conference addressed eight topical areas: superconductivity, materials for high-speed electronic devices, interfacial phenomena, amorphous materials, polymeric matrices in composites, metal and ceramic matrix composites, ceramics, and materials and devices processing. Highlights from the sessions are described below.

AMI is the materials research arm of the Colorado Advanced Technology Institute, a state agency created by the legislature as an economic development initiative involving industry, academia, and the state government. AMI's research agenda is totally industry driven, addressing the materials needs of its industry members through sponsored academic research. It functions as a consortium of nine corporations and Colorado's four research universities: (Colorado School of Mines as the lead institution), Colorado State University and the Universities of Colorado and Denver).

\section{Superconductivity I}

Chair: Robert Kamper, National Institute of Standards and Technology, Boulder, CO.

Alan F. Clark, recently returned from a year's assignment to the Office of Naval Research in London, opened the session with a keynote address on the superconductivity scene in Europe. He reminded the audience of the many major contributions that various European countries have made to the discovery and development of superconductivity. He reported a vigorous and coordinated effort to understand and develop high temperature superconductors, characterized by modest individual budgets but balanced by long-term commitment and a high degree of cooperation extending across national borders.

Five contributed papers focused on the fabrication of high temperature superconductors, reporting systematic investigations of the factors affecting various fabrication methods. Thin films, bulk ceramics and single crystals were all addressed. Fabrication methods included multilayer evaporation, single target sputtering and plasma spraying of thin films, hot isostatic pressing of bulk ceramics, and a chelating process for preparing starting material. One paper described the delicate art of growing single crystals of various cuprate superconductors.

\section{Superconductivity II}

Chair: Robert McConnell, Solar Energy Research Institute, Golden, $\mathrm{CO}$.

Six speakers presented samples of the state of the field. R.G. Eggert and J. Guzman opened the session, addressing the potential impact of high temperature superconductors (HTSC) on the mineral markets, especially those of copper and the rare earth metals. Although the magnitude of the impact is impossible to predict today, they said, it is possible to identify the ways mineral markets could be affected.

As testimony to the degree of progress in the field, two speakers addressed practical problems. B. Yarar et al. described a device that quantifies the Meissner effect, or the spontaneous expulsion of magnetic flux from the body of a superconducting material. An electronic signal is generated proportional to the repulsion which is counterbalanced by a feedback signal, which in turn is processed as a measure of the Meissner effect. The application is aimed toward quality control, possibly at the plant level. Another speaker, discussing magnetic suspension with HTSC, gave a theoretical derivation of suspension heights and dynamic response along with experimental results for three HTSC materials. A magnetic recording application was illustrated in which the recording head is suspended above the recording disk and never contacts the disk.

Two additional presentations dealt with more basic studies. One looked at the field and temperature dependence of the levitation properties and showed the existence of a threshold temperature above which the HTSC specimen will not levitate. The other reported experimental observations on the chemical modification of the insulator $\mathrm{YBa}_{2} \mathrm{Cu}_{3} \mathrm{O}_{6}$ to produce the HTSC $\left(\mathrm{Y}_{0.8} \mathrm{Ca}_{0.2}\right) \mathrm{Ba}_{2} \mathrm{Cu}_{3} \mathrm{O}_{6}$.

This session, like others on superconductivity, reflected the fast pace of progress in basic understanding and potential applications.

\section{Materials for High-Speed Electronic Devices I \\ Chair: Amoldo Majerfeld, University of Colo- mado, Boulder, $C O$.}

Presentations in this session covered a variety of materials of current interest for high-speed electronic and photonic devices. Important improvements in the purity control and reduction of the dislocation density in GaAs and Si substrates were reported. In the area of epitaxial III-V semiconductors, the astounding creativity demonstrated in artificially structured materials with nanometric dimensions attracted great interest. Keynote speaker V.G. Kerramidas discussed the use of such materials for optoelectronic integration, as well as the successful growth of epitaxial semi-metal/compound semiconductor novel materials. Several papers showed steady progress in the understanding of deep-level states in AlGaAs and the development of new optical characterization techniques for high purity GaAs and GaAs/AIGaAs interfaces.

\section{Materials for High-Speed Electronic Devices II}

Chair: Bruce Sabacky, Bandgap Technology Incorpomated, Broomfield, $\mathrm{CO}$.

A. Podell's keynote address was an entertaining as well as sobering assessment of the current market situation for devices and systems manufactured from highspeed electronic materials such as GaAs. Podell clearly outlined areas where materials producers need to substantially advance product quality, especially regarding uniformity of physical and electrical properties across a substrate. Well in tune with this keynote message, six authors presented work on methods to improve material and device performance. Their topics included new device structures, modeling techniques, and material processing techniques.

\section{Interfacial Phenomena I: Electronic Materials} Chair: Carl Wilmsen, Colomdo State University, Fort Collins, CO.

It is clear that the basic knowledge of surfaces and interfaces has advanced significantly the last few years. The basic tools of the past - Auger, x-ray and ultraviolet electron spectroscopy - have provided a rich base of information on the average properties of a surface. The introduction of the scanning tunneling microscope is now providing equally rich insight on the atomic level.

Pointed out in particular during the session was the important role of defects in initiating film growth and electronic state "pinning" on both Si and compound semiconductors. However, even with all the available research tools, many questions regarding gas/solid interactions on the solid surface remain unanswered - e.g., what is the physical cause of surface states on GaAs, how does $\mathrm{H}_{2}$ penetrate into a 
semiconductor, and how can epitaxy take place at low temperatures?

\section{Interfacial Phenomena II: Non-Electronic Materials}

Chair: Frank Schowengerdt, Colorado School of Mines, Golden, CO.

Papers in this session ran the gamut from advanced theory to applications, and included some novel characterization techniques. In the keynote presentation, J. Smith described an interesting universal relationship between binding energy and inter-particulate separation, which was shown to be valid from electron-hole liquids to nuclear matter. Other papers covered such topics as fracture behavior of $\mathrm{Cu}$ bicrystals (J. Wang et al.), prediction of interfacial bond strengths of $\mathrm{SiC} / \mathrm{Al}$ alloy composites (B. Lanning et al.), and plasma-enhanced chemical vapor deposition of thin diamond films (R.P.H. Chang et al.) Papers emphasizing interfacial characterization techniques included one on Auger plasmon energy losses (F.D. Schowengerdt et al.), and one on SEM/EDX characterization of rubber/metal interfaces (J. Ahn et al.) The session closed with a paper on corrosion and cracking of paint coatings on metallic surfaces of automobiles.

\section{Amorphous Materials I: \\ Semiconductors}

Chair: John Trefny, Colorado School of Mines, Golden, $\mathrm{CO}$.

The papers in this session were all of high quality and reflected a variety of interesting activities related to electronic amorphous materials. Keynote speaker Richard $S$. Crandall set an appropriate theme with his excellent overview of the StaeblerWronski effect and other metastabilities in amorphous silicon. These effects are not presently understood. Most of the succeeding authors referred to these metastabilities, either directly or indirectly. The second paper, by Michael Shur and coauthors, introduced some new concepts for a-Si thin film transition (TSTs). SERI/ CSM researchers followed with three papers on properties of a-Si doped materials and/or materials prepared by various deposition techniques. The final paper, by $\mathrm{K}$. Hong and R.F.Speyer of Alfred University, extended this theme to the II-IV-V $V_{2}$ semiconducting compounds.

\footnotetext{
Amorphous Materials II: Metals and Semiconductor Devices

Chair: Noboru Wada, Colorado School of Mines, Golden, CO.

This session dealt with three main subtopics: solid state reactions, amorphous me-
}

tallic alloys, and amorphous silicon devices. Metal atoms may diffuse through the interface of two adjacent crystalline metals, forming an amorphous phase at the boundary. A Cu-Y multilayer system showed that amorphization can occur even at room temperature, while an $\mathrm{Fe}-\mathrm{Zr}$ system demonstrated that solid state reaction can be induced by proton beam bombardment.

Because of their extremely high corrosion resistance and intriguing catalytic properties, intermetallic amorphous alloys have recently been investigated. Papers presented during this session focused on characterization and reactivity of various amorphous alloys.

Besides solar energy conversion applications, amorphous silicon can be used for many other devices. Developments of a spatial light modulator and a high-energy particle detector were discussed and appear to be promising new applications of this material.

\section{Polymeric Matrices in Composites}

Chair: John Stille, Colorado State University, Fort Collins, $\mathrm{CO}$.

Polymeric composites consisting of a matrix resin and a reinforcing component have many advantages over conventional materials for various end uses. Yet these materials have problems and room for improvement.

The most demanding properties are those required by the aerospace industry. As reviewed in K. Lau's keynote talk, composites with high strength at high temperatures are easy to fabricate and feature moderate costs. Recent years have seen several significant advances in this area. In conventional fiber-reinforced composites, the use of isoimide-imide conversion shows promise for a readily processable thermally stable polyimide. In addition, new curing systems are providing materials with higher thermal stability. An exciting emerging area involves the use of blends and the unconventional molecular composites. Molecular composites, which achieve reinforcement by a "molecular fiber" or rigid rod dissolved in the matrix resin, are showing very high strengths and thermal stabilities. In these systems, processability and lack of failure at the fiber matrix interface (because there is no interface) offer distinct advantages.

The most serious problems in conventional composites, such as failure at the fiber/matrix interface, moisture uptake, thermo-oxidative stability, mechanical durability at high use temperatures, were all addressed. A new method for forming silicon-carbide fibers from melt-spinnable polymer precursors holds promise for the production of high strength, thermally stable reinforcing materials.

\section{Metal and Ceramic Matrix Composites}

Chair: Jimmy Mote, University of Denver, Denver, $\mathrm{CO}$.

$\mathrm{S}$. Fishman's keynote paper reviewed research on metal and ceramic matrix composites at room temperature, and the next two papers covered research on the effects of elevated temperature on composites properties. In particular, the use of intermetallic compounds as high temperature matrix materials was explored by considering the phase stability and reaction kinetics of intermetallic/intermetallic composite systems.

R. Perkins' paper examined some oxidation-resistant aluminides reinforced with $\mathrm{SiC}$ or $\mathrm{TiB}_{2}$ as candidates for high temperature applications. W. Wu et al. addressed the erosion of $\mathrm{Al}$ and $\mathrm{Al} / \mathrm{SiC}$. P. Charalambides gave a detailed analytical approach to mixed mode delamination cracking in fiber-reinforced composites. The last two papers (J. Armstrong et al.) covered nondestructive methods for characterizing composites. K. Beasley and J. Ja$\mathrm{cob}$ et al. addressed thermal management by composites and composite processing economics, respectively.

The scope of the papers indicated that metal and ceramic matrix composites are serious contenders for many applications.

\section{Ceramics}

Chair: Gerald DePoorter, Colomado School of Mines, Golden, $\mathrm{CO}$.

Joel Katz (Los Alamos National Laboratory) started the session with a comprehensive keynote discussion of microwave sintering of ceramics. He discussed the history, theory, and practical applications of microwave sintering. Other discussions focused on the relationship of electronic properties to microwave heating, particularly regarding penetration depth (skin depth), and comparison of microwave and conventional heating.

S. Chiang was asked if any liquid phase was observed. He said none was detectable, although liquid phase should be seen at $1100^{\circ} \mathrm{C}$. He was also asked if the diffraction pattern changed from the shell to the core. Chiang responded that the shell was cubic and the core tetragonal.

Questions on paper J-5 focused on characterization of the $\mathrm{SiO}_{2}$ formed. M. Zachariah responded that not much characterization had been done because the measurements were made in situ.

Paper J-6 prompted questions on the 
sensitivity of the technique. R. Sato responded that the sensitivity is different for different nuclei, but there is no noticeable change for different environments of the same nuclei.

\section{Materials and Devices Processing}

Chair: Jon Johnston, Gates Rubber Company, Denver, CO.

A number of interesting papers were presented in this session, ranging over a wide variety of topics related to processing issues and prediction of material properties. Processing, processability, and the associated issues of material property prediction and control are important and often controlling issues in practical materials engineering. This session addressed these matters in the context of abrasion resistance in $\mathrm{TiC}$, cermet films, film resistors, conducting polymers, the future materials needs in electrophotographic processes, prediction of mechanical and piezoresistive properties of carbon fiber, rapid thermal annealing of thin films to control grain growth, processing to remove trace oxygen at increased removal efficiencies, and mechanical property degradation due to brazing of ceramic packages.

Jerome G. Morse AMI Director Research Professor of Physics Colorado School of Mines

Available Through MRS

Proceedings of the Industry-University Advanced Materials Conference II

Published by the

Advanced Materials Institute

Editor: F.W. Smith

1989, softcover, 98 papers, 888 pages

ISBN: 0-9624027-0-2

Order Code: AM-2B

$\$ 40.00$ MRS Members

$\$ 45.00$ U.S. List

$\$ 50.00$ Foreign

To order, call the MRS Publications

Department at (412) 367-3012;

fax (412) $367-4373$

For a free sample copy, write to:

Elsevier Science Publishers

P.O. Box 103

1000 AC Amsterdam, The Netherlands

\section{J.H. WERNICK}

Bell Communications Research, USA

\section{A.F.W. WILLOUGHBY}

The University of Southampton, U.K.

\section{MATERIALS LETTERS is the international forum for VERY FAST}

publication of timely results in the form of short, refereed papers. Thus, scientists are offered the opportunity to publish their new results rapidly.

MATERIALS LETTERS is an interdisciplinary journal, covering the area of materials science in its broadest sense, ranging from solid state physics to materials technology. Important topics include:

\section{* Preparation and Characterization of Materials \\ * High- $\mathrm{T}_{\mathrm{c}}$ Superconductivity \\ * Preparation, Characterization and Physics of Semiconductors \\ * Thin Films \\ * Physical Metallurgy, Mechanical Properties, Ceramics, Composites, etc.}

MATERIALS LETTERS has been affiliated with the Materials Research Society since the early years of the journal, and a significant part of the editorial board are MRS representatives.

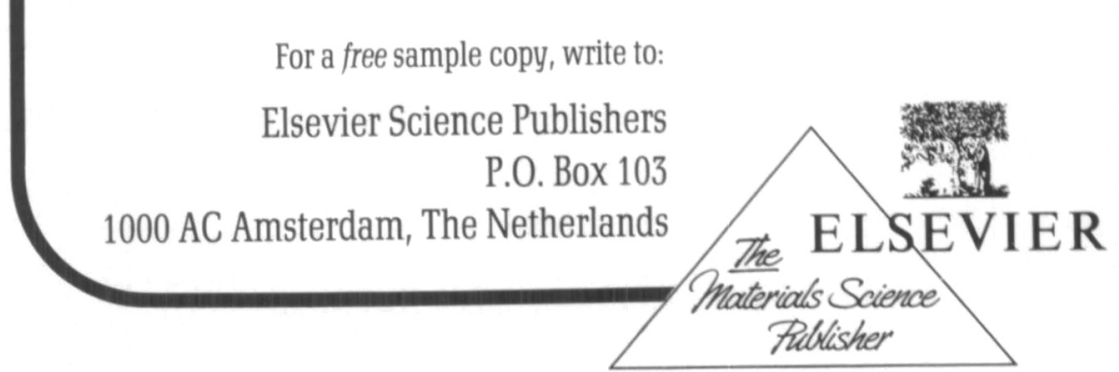

AMSTERDAM • LAUSANNE • LONDON • NEW YORK • OXFORD • TOKYO 